\title{
Human-like gait generation from a reduced set of tasks using the hierarchical control framework from robotics
}

\author{
Melya Boukheddimi ${ }^{1}$, François Bailly ${ }^{1,2}$, Philippe Souères ${ }^{1}$ and Bruno Watier ${ }^{1}$ \\ ${ }^{1}$ LAAS-CNRS, Université de Toulouse, CNRS, UPS, France \\ 7 Avenue du Colonel Roche, F-31400 Toulouse, France \\ ${ }^{2}$ Laboratoire de Simulation et Modélisation du Mouvement, Faculté de Médecine \\ Université de Montréal, Laval, QC, Canada \\ melya.boukheddimi@laas.fr
}

\begin{abstract}
This paper shows that controlling only a small set of adequately selected tasks is sufficient to closely reproduce the human gait kinematics. To this aim, a hierarchical controller is applied to a whole-body model including 42 degrees of freedom with 3 hierarchical tasks. The analysis of the simulated gaits shows the emergence of significant human-like properties in walking. In order to validate our results, a comparison between joint rotations in the simulated motion and in human reference motions is conducted. Finally, a discussion is given to illustrate the interest of the approach in view of related works.

Index Terms - Human gait simulation; Hierarchical control; Bipedal walking; Humanoid robotics.
\end{abstract}

\section{INTRODUCTION}

\section{A. Research significance}

The human body is a complex system made of more than 600 muscles, which contribute to the actuation of more than 200 Degrees of Freedom (DoFs) [1]. It is therefore a highly redundant system for most kinematic tasks. Many authors have suggested that the central nervous system does not independently control in real time each muscle and DoF [2]. Though the high number of muscles and DoFs make motor control problem difficult, it offers high adaptation capabilities to the body for executing multiple tasks simultaneously when necessary [2]. Among the tasks that require a high level of motor coordination, walking is a crucial one. The whole bipedal gait cycle is performed with two strides, each one including a double-stance phase and a swing phase. During the double-stance phase both feet remain in contact with the ground, whereas during the swing phase one limb oscillates from back to front [3]. Though this movement is quite stereotyped across individuals, it is still unclear how the central nervous coordinates the complex musculo-skeletal system, what is actually controlled and how the different sequences of the gait cycle are regulated.

\section{B. Related work}

To address such a challenge, most authors use simplified models of poly-articulated rigid bodies to represent the anatomical segments and the associated joints. These models include much less DoFs than the human body. Based on such

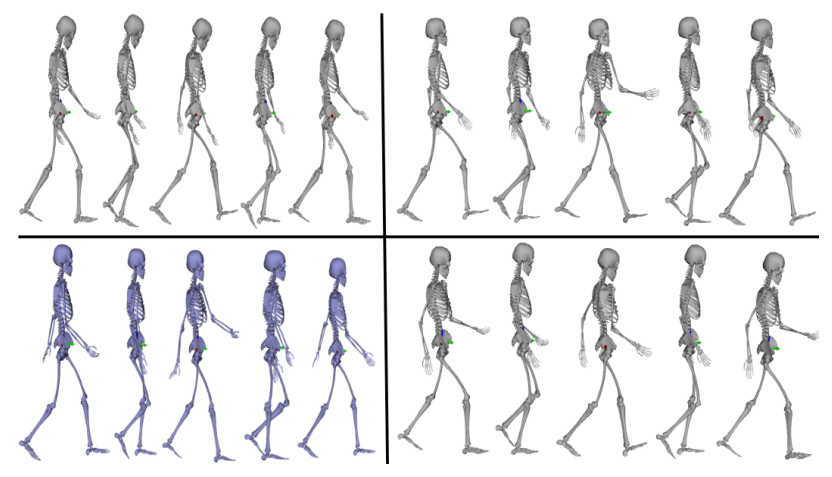

Fig. 1. Examples of human-like gaits obtained with hierarchical control.

models, four main approaches have been highlighted in the literature for gait generation: optimal control, inverse kinematics, reinforcement learning and hierarchical motor control. Optimal control consists in minimizing a cost function over a period of time. The cost is usually expressed in terms of physical parameters such as joint torques, jerk, kinetic energy or the distance between the current and the desired position. In [4] optimal control with direct multiple-shooting was used to produce gait with a 34-DoFs robot model. Several optimization criteria were tested along a single swing of five phases, with different contact constraints, and the obtained motion was compared to captured human movements. Inverse kinematics is commonly used to determine a coordination of joint rotations that leads to an expected body displacement, based on the equations of the kinematics. It was used in [5] to simulate whole-body walking, using a model based on OpenSim to actuate the lower limbs and considering as input actual human movements provided by motion capture (MoCap). Reinforcement learning is a very active and innovative field which was also used for bipedal gait simulation. In [6] no reward-based guidance was required and robust locomotion behaviours emerged from distributed proximal policy optimization. Finally, hierarchical motor control consists in generating movements by executing a stack of tasks with priority order. In [7] a strict priority optimization was used 
to reproduce locomotion by considering the tasks as physical properties, like end-effector position or angular momentum. The authors showed that their controller was robust to the modifications of the body parameters.

\section{Proposed solution}

In this paper, we use hierarchical motor control for generating gait. Instead of tackling the dynamical problem, our aim is to demonstrate that the prioritized regulation of a small set of kinematic tasks is sufficient to coordinate the joints of 42-DoFs model in order to closely reproduce a humanlike gait. Our work is based on a careful observation of human walking patterns from which we have identified a set of ordered tasks that are representative of the kinematic gait organization. In order to represent the control in a solid mathematical framework we use the task-function formalism developed by roboticists [8]. We demonstrate the consistency of our approach by comparing the simulated joint rotations obtained by our method with experimental human walking patterns given by MoCap. The paper is organized as follows. A presentation of the model and of the experimental data used in this work is given in Sec. II. Section III recalls the task function formalism that we used for motion generation. Our gait simulation results are presented in Sec. IV. The simulated gaits are compared with the human reference in Sec. V. Finally Sec. VI provides a discussion about the interest of the approach.

\section{GAIT ANALYSIS AND RECONSTRUCTION}

\section{A. Data}

Experiments were conducted in our laboratory with the aim to record a database of human gait cycles. Each participant performed at least 3 trials of normal gait cycles at spontaneous speed, one cycle per participant was used. The volunteers were nine healthy men, (age: $24.2 \pm 2.3$ yr, height: $1.74 \pm 0.09 \mathrm{~m}$, mass: $71.0 \pm 9.0 \mathrm{~kg}$ ). The experimental setting was equipped with 13 infrared cameras sampling at $200 \mathrm{~Hz}$ (Vicon, Oxford Metrics, UK), which recorded the motions of 43 reflective markers attached to the participants body [9]. The markers set was placed in accordance with the International Society of Biomechanics (ISB) and $\mathrm{Wu}$ guidelines [10]. The data were extracted using the adjusted scaling equations from anthropomorphic relationships from [11].

\section{B. Model}

The same simplified whole-body 3D skeletal model [12] was used for human gait reconstruction and for the simulation. This model is based on the OpenSim musculo-skeletal model [13], which includes $n=42$ DoFs and $n_{s}=19$ segments, as depicted in Fig. 2. Using these 42 DoFs main features of complex human movements such as whole-body walking or Parkour motions can be closely reproduced, as

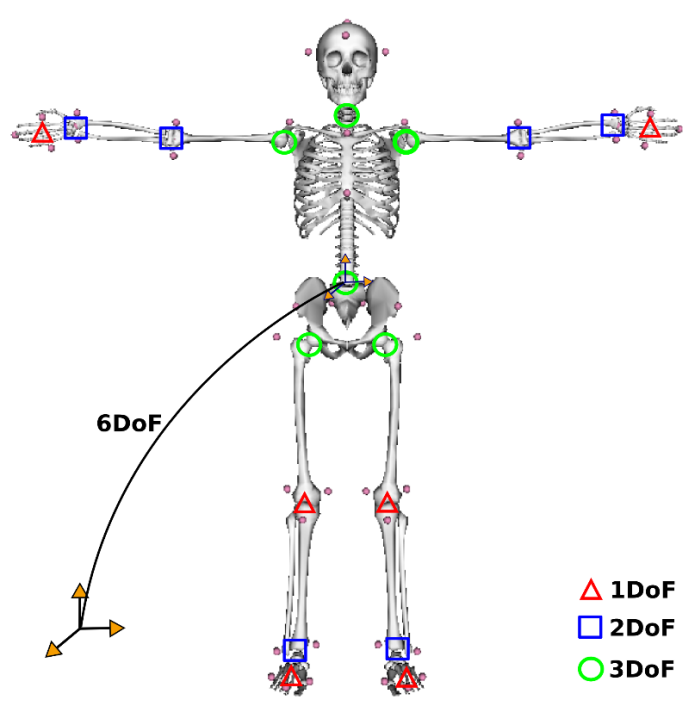

Fig. 2. Whole-body 3D skeletal model [12]

shown in [12]. The model, parametrized with frictionless articulated multi-body dynamic, includes:

- 8 DoFs in each upper limb (3 at the shoulder, 2 at the elbow, 2 at the wrist, 1 at the hand),

- 7 DoFs in each lower limb (3 at the hip, 1 at the knee, 2 at the ankle, 1 at the foot),

- 6 DoFs to describe the position (3DoFs) and the orientation (3DoFs) of the pelvis, modeled as free-flyer,

- 3 DoFs at the lower back,

- 3 DoFs at the neck.

The skeletal models for the nine subjects were scaled to each participant height using the OpenSim Software [13].

\section{Whole-body kinematics computation}

The whole-body gait kinematics was reconstructed from MoCap, using the Inverse Kinematics (IK) OpenSim Software [13]. Joints angles were deduced from the resolution of the least-squared problem (1), using a general-purpose quadratic programming $(\mathrm{QP})$ solver:

$$
\begin{gathered}
\min _{q} \sum_{i=1}^{N_{m}} w_{i}\left\|x_{i}^{e x p}-x_{i}(q)\right\|^{2} \\
\text { s.t. } \quad \underline{q} \leq q \leq \bar{q}
\end{gathered}
$$

where:

- $N_{m}=43$ is the total number of markers.

- $q \in \mathbf{R}^{n}$ is the vector of generalized coordinates which parameterizes the body configuration. It includes $n-6$ variables to describe the value of joint angles, plus 6 variables to describe the position and orientation of a reference frame attached to the pelvis, usually referred to as the "root frame".

- $\underline{q}$ and $\bar{q}$ are the minimum and maximum bounds of the generalized coordinates. For the $n-6$ variables that 
describe the joint angles, these bounds represent the natural joint limits as in [12], whereas for the remaining 6 variables, these bounds are used to limit the region inside which the pelvis is located, for computational purposes.

- $x_{i}^{\text {exp }}$ is the experimentally-measured position of the $i^{\text {th }}$ marker, $1 \leq i \leq N_{m}$.

- $x_{i}(q)$ is the position of the $i^{t h}$ marker deduced from the model.

- $w_{i}$ is the weight associated to the $i^{t h}$ marker in the optimization process.

The generalized coordinates solutions of (1) constitute our "reference trajectories". They will be used as ground truth values to evaluate the quality of the simulated gaits in Sec. V.

\section{THE TASK FUNCTION FORMALISM}

In order to simulate the gait kinematics, we have chosen to rely on the task-function formalism used in robotics [8], [14] [15]. This approach aims at computing the body movements by regulating to zero a set of specific output functions, called task-functions, following a prescribed hierarchic order. A task function $e(q) \in \mathbb{R}^{m}$ is defined as the difference between a vector-valued function of the generalized body coordinates $f(q) \in \mathbb{R}^{m}$ and a reference vector $f^{*} \in \mathbb{R}^{m}$, namely: $e(q)=$ $f(q)-f^{*}$. The task function represents the gap that needs to be brought to zero for the tasks to be accomplished. For instance, a task-function $e_{\text {com }}(q)=c(q)-c^{*}$ can be used to move the center of mass (CoM), from its current position $c(q) \in \mathbb{R}^{3}$, to a target position $c^{*} \in \mathbb{R}^{3}$, or to follow a given trajectory $c^{*}(t) \in \mathbb{R}^{3}$. In order to overcome this gap, a body motion $q(t)$ has to be executed. During the movement, the time-derivative of the task-function is related to the body model velocity $\dot{q}$ by the task Jacobian $J_{e}(q)=\partial e / \partial q$, as expressed by (2):

$$
\dot{e}=\frac{\partial e}{\partial q} \frac{d q}{d t}=J_{e} \dot{q}
$$

As $m \leq n$, the system is usually redundant with respect to the task. As a consequence an infinite number of motions are suitable to achieve the task. Among them, the minimal norm solution can be obtained by using the pseudo inverse $J_{e}^{+}=J_{e}^{T}\left(J_{e} J_{e}^{T}\right)^{-1}$ of the task Jacobian $J_{e}$ :

$$
\dot{q}=J_{e}^{+} \dot{e}
$$

Indeed, it is a well known result that (3) provides the optimal solution of the quadratic programming (QP) problem :

$$
\min _{\dot{q}}\left\|J_{e} \dot{q}-\dot{e}\right\|^{2}
$$

Other movements, which provide the same guarantee for the execution of the task, can be obtained by adding to (3) any element of the null space of $J_{e}$ as follows:

$$
\dot{q}=J_{e}^{+} \dot{e}+P_{e} g_{s}
$$

where $P_{e}=\left(I_{n}-J_{e}^{+} J_{e}\right)$ is the projector onto the null space of $J_{e}$, and $g_{s} \in \mathbb{R}^{n}$ is an added velocity component that can be used to execute a secondary task. More precisely, consider that $e$ is the task to be executed with the highest priority, while $e_{s}$ is a secondary task, associated to the Jacobian $J_{e_{s}}$, to be executed at best using the unconstrained DoFs. In that case, introducing the expression of $\dot{q}$ given by (5) into the dynamics of the lower priority task leads to:

$$
\dot{e}_{s}=J_{e_{s}} \dot{q}=J_{e_{s}} J_{e}^{+} \dot{e}+J_{e_{s}} P_{e} g_{s}
$$

Then, solving this equation in $g_{s}$ yields:

$$
g_{s}=\left(J_{e_{s}} P_{e}\right)^{+}\left(\dot{e}_{s}-J_{e_{s}} J_{e}^{+} \dot{e}\right)
$$

Finally, injecting (7) in (5) leads to the expression of the instantaneous generalized velocity of the body model to execute tasks $e$ and $e_{s}$ with the prescribed priority order:

$$
\dot{q}=J_{e}^{+} \dot{e}+P_{e}\left(J_{e_{s}} P_{e}\right)^{+}\left(\dot{e}_{s}-J_{e_{s}} J_{e}^{+} \dot{e}\right)
$$

To close the loop, a decay rate of the task functions has to be chosen. Classically a second order dynamics of the type $\ddot{e}=-\lambda_{p} e-\lambda_{d} \dot{e}$ with appropriate proportional and derivative gains, $\lambda_{p}$ and $\lambda_{d}$, is used to ensure the convergence of task function to zero with the expected behavior, as in [16]. To this end, the link between the second-order derivative of the task function and the acceleration of the generalized coordinates is obtained by differentiating once more (2) as follows:

$$
\ddot{e}=J_{e} \ddot{q}+\dot{J}_{e} \dot{q}
$$

As demonstrated in [17], the control scheme (8) can be iteratively extended to cope with any arbitrary sequence of tasks with priority order, provided that sufficiently many DoFs are available. Each task is to be executed under the constraint that the task functions of higher priority are regulated to zero. This comes to define an iterative optimization problem under equality constraints. The main limitation of this iterative process is that it does not allow to cope with inequality constraints. Fortunately, as each step of the process is equivalent to solving a QP problem (4), a global solution can be obtained by using a numerical hierarchical quadratic programming (HQP) solver to cope in cascade with both equality and inequality constraints [18], [19]. In this paper we use the HQP solver developed in [20] for synthesizing walking motions based on a hierarchy of tasks.

In our work, we use the non-penetration contact constraints [21]. During walking, the points that are in contact with the ground remain motionless. Therefore, the velocity of the contact points is equal to zero, $v_{c}=0$, during $\Delta t_{c}$. Hence the acceleration at this contact point is zero, $a_{c}=0$.

\section{GAit SIMULATION}

This section introduces the gait decomposition and the task hierarchy that were used for the simulation. 


\section{A. Decomposition of the gait}

It is a well-known fact that the human gait includes alternating contact configurations of the feet with singlesupport and double-support phases, as shown in Fig. 3. Single support is when only one foot is in contact with the ground. It starts just after the toes of the swinging foot take off, and ends just before the heel of the swinging foot lands on the ground. Double support is when both feet are in contact with the ground. It starts when the heel of the swinging foot lands on the ground, and ends when the toes of the next swinging foot take off.

\section{B. Hierarchy hypothesis}

Based on the observations of the human gait and the experimentatal tests we conducted with the hierarchical solver, the following features were identified as key elements for the simulation:

1) The three-dimensional trajectory of the whole-body CoM is crucial for gait simulation [22]. Indeed, based on this reference trajectory the hierarchical solver is able to determine the trajectories of different body segments, notably the height of the upper limbs of the body and the height of the feet.

2) The trajectory of the foot in the horizontal plane is necessary to reconstruct its roll motion. As this information cannot be precisely computed by the hierarchical solver from the three-dimensional trajectory of the CoM because of their relative mass, it must be specified as input.

3) In order to cope with redundancy and to closely mimic the human reference, an additional low priority joint posture task needs to be added. Tracking the reference joint configuration at the end of each phase seems to be an efficient solution.

Based on these observations, the hierarchy of task depicted in Fig. 3 was designed and ordered as follows :

- Highest priority task: tracking of the heel reference trajectory of the swinging foot in the horizontal plane $(x, y)$ and positioning of the heel in contact with the ground in the horizontal plane $(x, y)$ (along the antheropostero and medio-lateral axis).

- Second priority task: tracking of the reference threedimensional trajectory whole-body $\operatorname{CoM}(x, y, z)$.

- Third priority task: minimization of the gap between the current body joint configuration and the reference joint configuration, at the end of each phase, depicted as a fixed vector of dimension $1 \times 42$.

This hierarchy was applied to each phase of the movement. We obtained nine simulations of complete cycles of gait, using the nine scaled whole-body models. The videos of the gaits are available online here: https://cloud.laas.fr/index.php/ s/EHGzcT9uXtIPOBj. Fig. 1 presents sequences of snapshots of generated gaits cycle, and the full gaits can be observed in

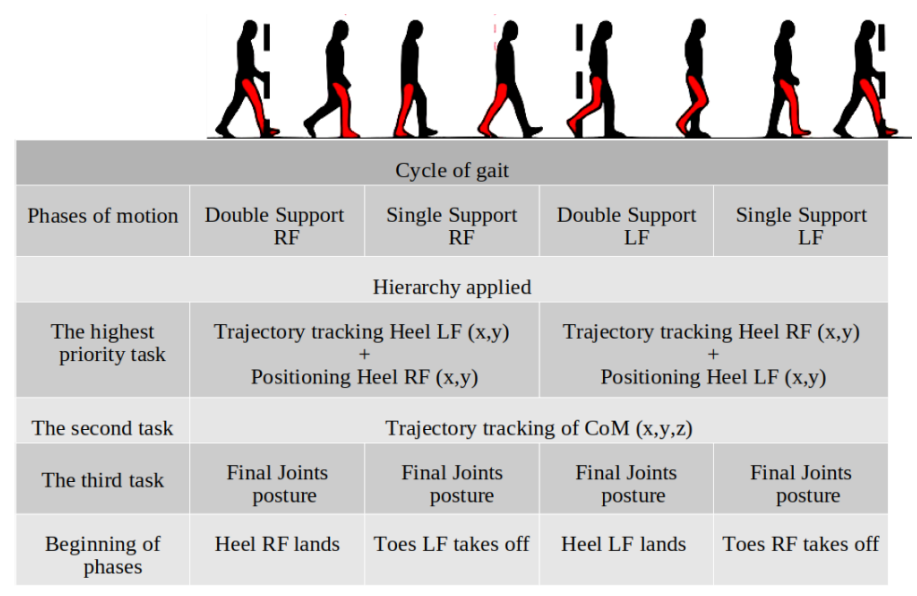

Fig. 3. Task hierarchy along the successive phases of the gait cycle. RF: right foot, LF: left foot.

the attached videos. A first visual analysis of the simulated gaits reveals some relevant human walking properties that are usually difficult to obtain in robotics. In particular the simulated motions show a good flexion and extension of the swinging knee and hip with a natural hip-swaying. It is also interesting to observe that a natural roll of the foot with the ground is performed. The upper part of the body including the head follows a human profile, and the back and forth movement of the arms is well reproduced. Beyond this first visual observations, a more careful analysis of the simulated gaits is provided in the next section. The simulated gaits are compared with the human reference based on biomechanical criteria.

\section{COMPARISON}

The following criteria, which are commonly used in biomechanics, were used for our comparative study:

- Range Of Motion (ROMs) of the joint rotations.

- Mean and standard deviation.

- Student test $(\alpha=0.05)$.

- Shapiro-Wilk test of normality $(\alpha=0.05)$. This assesses the normality of the data which are a prerequisite for the Student test.

Fig. 4 to 7 show the means and standard deviations over one gait cycle and for the nine simulated gaits and the reference human movements. Due to space limitation, only the most significant joint rotations involved in walking are presented. Table I reports, for both the reference and the simulation, the mean ROM values (in degrees) and their differences (errors) of the most relevant joint rotations over the nine walking subjects. One can notice that the error values are always under 15 degrees and often below 5 degrees. The last column of Table I indicates the significance of the Student test. This indicator shows that the simulated flexion-extension of the hip (right, left) and the simulated abduction-adduction of 


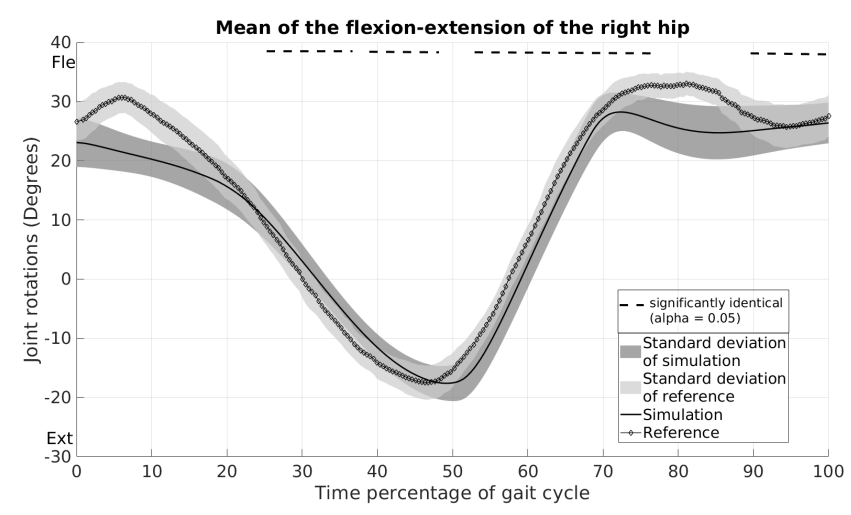

Fig. 4. Mean of the flexion-extension of the right hip

TABLE I

THE MEANS OF ROMS FOR THE JOINT ROTATIONS AND THEIR DIFFERENCES (DEGREES), THE STUDENT TEST, $\alpha=0.05 . \ddagger$ SIGNIFICANTLY IDENTICAL. R : RIGHT, L : LEFT.

\begin{tabular}{|l|c|c|c|}
\hline \multicolumn{1}{|c|}{ Joint Rotations } & $\begin{array}{c}\text { ROMs } \\
\text { Simulation }\end{array}$ & $\begin{array}{c}\text { ROMs } \\
\text { Reference }\end{array}$ & $\begin{array}{c}\text { ROMs } \\
\text { Difference }\end{array}$ \\
\hline HIP R Flex/Exte & 52 & 54 & $2 \ddagger$ \\
\hline HIP R Abdu/Aduc & 22 & 24 & $2 \ddagger$ \\
\hline HIP R Axial rotation & 11 & 16 & 5 \\
\hline Knee R Flex/Exte & 56 & 67 & 11 \\
\hline Ankle R Dors/Plan & 20 & 34 & 14 \\
\hline Ankle R Ever/Inve & 23 & 27 & 4 \\
\hline Hip L Flex/Exte & 57 & 57 & $0 \ddagger$ \\
\hline Hip L Abdu/Aduc & 23 & 26 & 3 \\
\hline Hip L Axial rotation & 13 & 16 & 9 \\
\hline Knee L Flex/Exte & 54 & 66 & 13 \\
\hline Ankle L Dors/Plan & 21 & 35 & 4 \\
\hline Ankle L Ever/Inve & 24 & 28 & 3 \\
\hline
\end{tabular}

the right hip are significantly identical to the reference. The results demonstrate that the simulated joint rotations closely follow the human reference. The joint rotations do not always reach the maximal rotations made by the reference. However, the ROM remain always equal or at lower value compared to the reference; this might reflect the low priority of the body joint configuration task.

\section{DISCUSSION}

The results presented in the previous section confirm the assumptions made in Sec. IV-B, namely that the human gait kinematics can be closely reproduced from a reduced number of motor tasks using a hierarchical controller. In accordance with the theory of human motor control it shows that complex motions, like walking can be obtained from the control of a few number of primitive elements. It is also interesting to notice that the simulated gaits were obtained without constraining the height of the feet. In this work a particular attention was given to the choice of the tasks, their

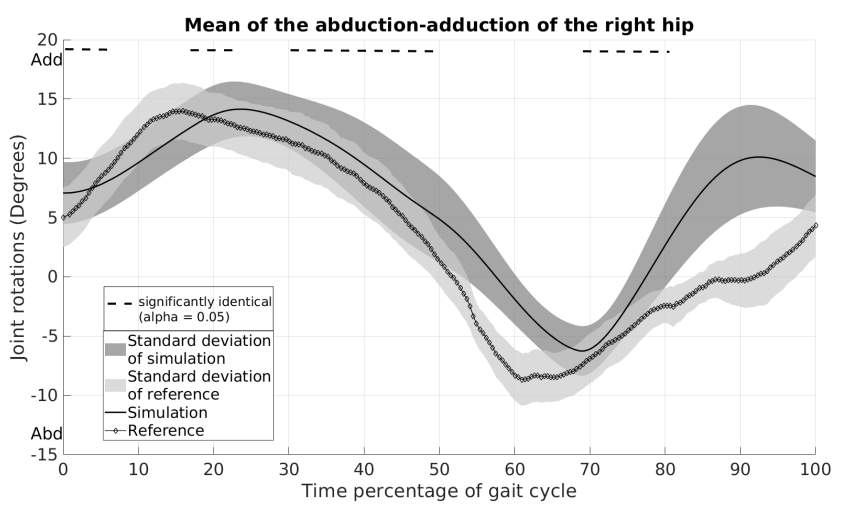

Fig. 5. Mean of the abduction-adduction of the right hip

priority order and the decomposition of the gait cycle. The choice of this decomposition was based on biomechanical observations of the normalized human gait cycle. Only 4 phases were sufficient to describe the whole cycle in our study, whereas more complex decompositions were necessary in other simulation approaches. Indeed, the gait cycle was divided into 8 different phases in [23], and 8 different phases were used in [7]. In [4], a half gait cycle was generated using optimal control, dividing the swing into 5 phases. We tried to consider a gait decomposition based on two phases only, but the simulation resulted in an abnormal gait with a straight knee for the swinging foot. Our contribution is also in the identification of key minimal criteria for the generation of an anthropomorphic gait, with only 3 ordered tasks for each phase. Indeed, different kinds of criteria were proposed in the literature using usually more complex schemes to simulate walking. In [7], 8 different tasks were used to generate gait, and the three-dimensional trajectory of the whole-body CoM was separated into two different tasks components in the horizontal and the sagittal planes. In our experiments, we noticed better results without such a separation. Furthermore the reduced number of key criteria is a way to introduce more motor variability, which is crucial for multiple tasks execution, according to the theory of motor abundance [2].

\section{CONCLUSION}

Based on the analysis of human waking a reduced set of key kinematic criteria was identified and used for generating human-like gait with a poly-articulated model, using the hierarchical control framework from robotics. A 3D wholebody model including 42 DoFs was used both for reconstructing the human motion for motion capture measurements and for simulation. Nine different gaits were simulated by considering a decomposition of the gait cycle into four phases and three ordered tasks consistently parameterized for each phase. The simulated results were analyzed and compared with the human reference, according to a set of biomechanical criteria. This comparative analysis showed that the simulated 


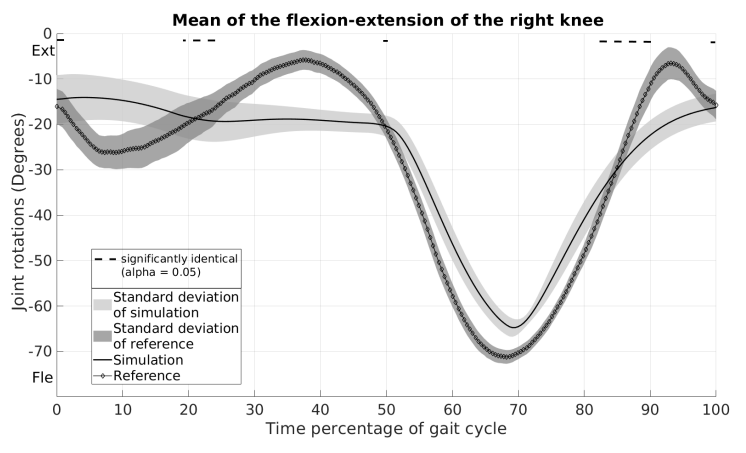

Fig. 6. Mean of the flexion-extension of the right knee

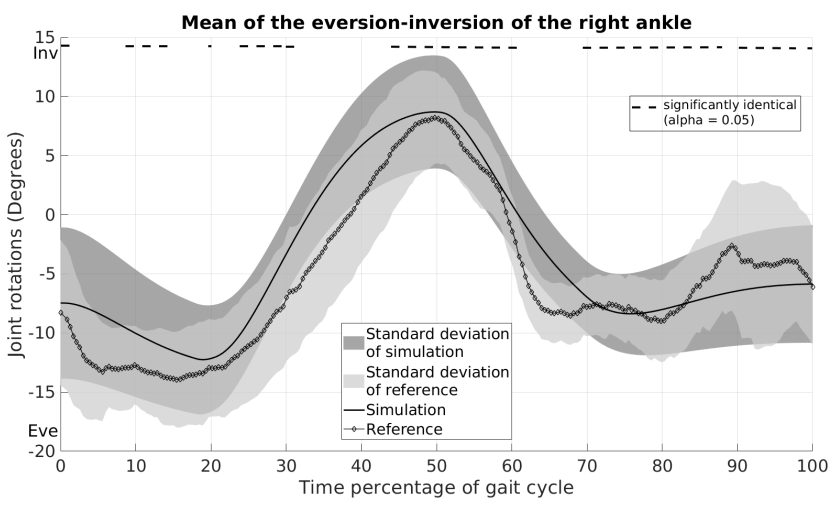

Fig. 7. Mean of the eversion-inversion of the right ankle

gaits closely follow the human gait kinematics. Though this study only considered the motion at the kinematic level, it shows that the coordination of a complex poly-articulated system can be deduced from a reduced number of criteria, notably in the case of cyclic movements. Obviously, in order to guarantee the feasibility of the motion, the wholebody dynamics needs be considered and the control must be expressed in terms of joint torques and contact forces. This constitutes the next challenging step of our study.

\section{ACKNOWLEDGMENT}

This work was partially supported by the European project RoboCom++, and by the French National Research agency (ANR), through the projects ANR-HOBIS (18CE27- 0010) and ANR-COBOT (18CE10 - 0003).

\section{REFERENCES}

[1] D. Fontaine, D. David, and Y. Caritu, "Sourceless human body motion capture," 012003.

[2] M. L. Latash, J. P. Scholz, and G. Schöner, "Motor control strategies revealed in the structure of motor variability," Exercise and sport sciences reviews, vol. 30, no. 1, pp. 26-31, 2002.

[3] E. Viel, La marche humaine, la course et le saut: biomécanique, explorations, normes et dysfonctionnements, ser. Le Point en rééducation. Masson, 2000.

[4] M. L. Felis and K. Mombaur, "Synthesis of full-body 3-d human gait using optimal control methods," in 2016 IEEE (ICRA), May 2016, pp. $1560-1566$
[5] A. Rajagopal, C. L. Dembia, M. S. DeMers, D. D. Delp, J. L. Hicks, and S. L. Delp, "Full-body musculoskeletal model for muscledriven simulation of human gait," IEEE Transactions on Biomedical Engineering, vol. 63, no. 10, pp. 2068-2079, Oct 2016.

[6] N. Heess, D. TB, S. Sriram, J. Lemmon, J. Merel, G. Wayne, Y. Tassa, T. Erez, Z. Wang, S. M. A. Eslami, M. A. Riedmiller, and D. Silver, "Emergence of locomotion behaviours in rich environments," CoRR, vol. abs/1707.02286, 2017

[7] M. de Lasa, I. Mordatch, and A. Hertzmann, "Feature-Based Locomotion Controllers," ACM Transactions on Graphics, vol. 29, no. 3, 2010

[8] C. Samson, M. Leborgne, and B. Espiau, Robot Control; The Task Function Approach, ser. Oxford Engineering Science Series 22. Oxford Science Publications, 1991.

[9] J. Carpentier, M. Benallegue, and J.-P. Laumond, "On the centre of mass motion in human walking," Int. J. Autom. Comput., vol. 14, no. 5, pp. 542-551, Oct. 2017.

[10] G. Wu, F. van der Helm, H. Veeger, M. Makhsous, P. van Roy, C. Anglin, J. Nagels, A. Karduna, K. McQuade, X. Wang, F. Werner, and B. Buchholz, "Isb recommendation on definitions of joint coordinate systems of various joints for the reporting of human joint motion-part ii: shoulder, elbow, wrist and hand," Jr of Biomechanics, vol. 38, pp. 981-92, 2005.

[11] R. Dumas, L. Cheze, and J.-P. Verriest, "Adjustments to mcconville et al. and young et al. body segment inertial parameters," Journal of Biomechanics, vol. 40, no. 3, pp. 543 - 553, 2007.

[12] G. Maldonado, P. Souères, and B. Watier, "Whole-body musculoskeletal model v1," Université de Toulouse 3 Paul Sabatier ; LAASCNRS, Technical Report Rapport LAAS n 18233, 2018.

[13] S. Delp, F. Anderson, A. Arnold, P. Loan, A. Habib, C. John, E. Guendelman, and D. Thelen, "Opensim: Open-source software to create and analyze dynamic simulations of movement," Biomedical Engineering, IEEE Transactions on, vol. 54, pp. 1940 - 1950, 122007.

[14] L. Saab, N. Mansard, F. Keith, J.-Y. Fourquet, and P. Souères, "Generation of dynamic motion for anthropomorphic systems under prioritized equality and inequality constraints," in 2011 IEEE ICRA 2011, Shanghai, China, May 2011, pp. pp. 1091-1096.

[15] N. Mansard, O. Stasse, P. Evrard, and A. Kheddar, "A versatile generalized inverted kinematics implementation for collaborative working humanoid robots: the Stack of Tasks," in ICAR'09, Munich, Germany, Jun. 2009, pp. 1-6.

[16] L. Saab, O. E. Ramos Ponce, F. Keith, N. Mansard, P. Souères, and J.Y. Fourquet, "Dynamic Whole-Body Motion Generation Under Rigid Contacts and Other Unilateral Constraints," IEEE Transactions on Robotics, vol. 29, no. 2, pp. 346-362, Apr. 2013.

[17] B. Siciliano and J. J. Slotine, "A general framework for managing multiple tasks in highly redundant robotic systems," Fifth International Conference on Advanced Robotics 'Robots in Unstructured Environments, pp. 1211-1216 vol.2, 1991.

[18] O. Kanoun, F. Lamiraux, P.-B. Wieber, F. Kanehiro, E. Yoshida, and J.-P. Laumond, "Prioritizing linear equality and inequality systems: application to local motion planning for redundant robots," in ICRA 2009 - IEEE, Kobe, Japan, May 2009, pp. 2939-2944.

[19] A. Escande, N. Mansard, and P.-B. Wieber, "Fast Resolution of Hierarchized Inverse Kinematics with Inequality Constraints," in ICRA 2010 - IEEE, Anchorage, United States, May 2010, pp. 3733-3738.

[20] A. Escande, N. Mansard, and P. Wieber, "Hierarchical quadratic programming: Fast online humanoid-robot motion generation," The International Jr of Robotics Research, vol. 33, no. 7, pp. 1006-1028, 2014

[21] D. Baraff, "Non-penetrating rigid body simulation," in SIGGRAPH 95 Course Note 34. ACM SIGGRAPH, 1993.

[22] J.-P. Laumond, M. Benallegue, J. Carpentier, and A. Berthoz, "The Yoyo-Man," The International Jr of Robotics Research, vol. 36, no. 13-14, pp. 1508 - 1520, Dec. 2017.

[23] C. D. Joshi, U. Lahiri, and N. V. Thakor, "Classification of gait phases from lower limb emg: Application to exoskeleton orthosis," 2013 IEEE Point-of-Care Healthcare Technologies (PHT), pp. 228-231, 2013. 